\title{
L'épanouissement de la Lumière dans "La Semaison" dePhilippe Jacottet
}

\author{
Dr. Siham Abd El Fattah Mohamed Gabr \\ Professeur adjoint de la littérature française
}

Faculté des lettres

Université de ZAGAZIG

\section{Introduction}

Dans notre modeste recherche qui évoque le thème de la Lumière dans "La Semaison" de Jacottet, sorte de prose poétique; nous tenterons d'aborder un sujet qui nous fascine, nous attire et nous intrigue.

L'intérêt que voue le poète à la Lumière outre le sens de la mesure en raison d'une sensibilité particulièrement excessive doublée d'humilité.

Nous tenterons d'accompagner Jacottet le long de son parcours analytique qui va de l'essence même des choses jusqu'à l'épanouissement de cette Lumière qui les anime avec tout le scintillement de son chatoiement.

En dépit du trait succint de notre recherche, elle englobera l'ampleur de l'énergie qui dispense cette lumière perçue sous sa forme apparente de vitalité et de dynamisme. Ce modeste ouvrage essaiera dans la limite du possible de 
démontrer l'importance incontournable de cette source d'énergie sans laquelle la vie devient une sorte d'exposition de potiches.

Nous espérons que nos lecteurs retrouveront dans cet essai le stimulus d'une profonde méditation qui les incitera à découvrir davantage le fabuleux qui entoure le rayonnement de cette lumière pour profiter à fond de son caractère utile et agréable.

\section{I- NATURE de cette LUMIÉRE}

\section{A) L'insaisissable :}

Un des traits caractéristiques de la Lumière c'est qu'elle est insaisissable. Sa vitesse considérable lui a valu ce qualificatif. Jacottet souligne ce trait spécifique de la Lumière en évoquant une citation de Phédon :

«Comme s'il fallait de toute nécessité qu'un point restât douteux éternellement, échappât aux prises de la lumière fuyante.» 1

Ce trait particulièrement insaisissable est bien décrit dans une formule parfaitement révélatrice. Celle de la forêt où Jacottet retrouve la source même de l'hospitalité, de l'amitié. Observons cet accueil chaleureux de la forêt sous l'égide d'une lumière douce, souple, euphorique mais d'une fugacité surprenante : 
«La maison ouverte, la forêt est une maison aux fenêtres, aux portes ouvertes. La lumière y circule comme dans les rues. Passe, entre et sort. La lumière ou la rêverie dévêtue, celle qu'on ne rattrapera plus.» ${ }^{2}$

Jaccottet en tant que poète qui jouit d'une puissante sensibilité ressent physiquement la présence de cette lumière invisible, telle que la définit les scientifiques dans le Petit Larousse : "Des ondes électromagnétiques. Des ondes qui jouissent de leur facultés : celle de l'électricité d'éclairer et du magnetisme "d'aimanter" ou "d'attirer" comme le décrit Jaccottet poétiquement pour expliquer l'effet lumineux de l'intuition considérée comme une énigme par les profanes :

«N'est-ce pas le fait d'un esprit borné qui refuse de croire à l'énigme qui nous attire et nous éclaire.» ${ }^{3}$

Sa description n'en demeure pas moins une véritable confirmation qui mérite le titre d'une "expérience" quasi scientifique :

«Il s'agit là, évidemment d'une expérience, d'un fait indubitable.» 4

Une expérience, à l'instar de celles réalisées dans un laboratoire, dont seul le poète peut ressentir les effets approuvés par un résultat probant :

«Le résultat est toujours le même.» ${ }^{5}$ 
Plus d'une génération ont confirme avec le poète l'authenticité de ses résultats :

«Elle s'est produite depuis qu'il y a des hommes.» ${ }^{6}$

\section{B) Son Caractère Abstrait :}

Cette lumière fait partie du quotidien, qui nous anime, nous imprègne de sa chaleur bénéfique. Elle traverse tout le règne végétal et animal sur terre, et lui inculque son dynamisme reflétant le mouvement et l'énergie vitale qui préserve et maintient la vie sur terre.

Le poète observe particulièrement dans cette lumière vu le côté purement abstrait : le Temps - la Durée - Quand l'énergie de cette lumière s'accentue et sa puissance s'amplifie sa douceur se convertit en une étincelle éblouissante. Dans une image simple, imprégnée de sens et de symbolisme le réalisme et le merveilleux se conjuguent en une symbiose harmonieuse entre l'abstrait et le concret. Jaccottet décrit cette alchimie subtile de l'effort humain à travers le Temps ou la Lumière dans un moment où la fatigue devient étincelle :

\section{«Ainsi le temps nous use et nos travaux étincellent un instant sous ces coups. $\rangle^{7}$}

L'ampleur de cet imaginaire s'accentue quand le poète affronte la vie sur terre à la mort à l'instar du jour et de la nuit dans le cycle de notre vie quotidienne. 
Observons de près cette comparaison exceptionnelle à la fin de la journée quand la nature commence à épouser la nuit. Le meilleur support pour matérialiser ces grandes valeurs abstraites du sens de la durée et du temps chez Jaccottet c'est la neige. Le grand "réservoir" de lumière : le feu même qui anime la vie commence à s'épuiser et traîne :

«Traînées de feu dans l'herbe avant la neige comme ce flamboiement au ciel d'ouest avant la nuit. Sursaut de l'âme avant la mort. ${ }^{8}$

A la fin de la journée c'est le relais : la nuit continue le rythme du jour comme l'invisible dont la face apparente est l'obscurité prend le relais ou visible.

Jaccottet l'adepte inconditionnel de la lumière retrouve en elle une puissance dynamique qui charrie la vie dans ses veines, et que l'on appelle familièrement dans le langage médical "la lumière des artères" celle qui charrie de flux du sang dans les veines.

Le poète dans les moments de verve poétique observe dans l'obscurité qui enveloppe son poing une irisation puissante. Et la main revêt alors l'image matérielle d'une "torche" qui par sa puissante lumière dissipe dans l'âme du poète le sens négatif de l'obscurité. Grâce à cette "torche" la face apparente de l'invisible apparaît fort agréable et l'obscurité n'impressionne plus le poète. 
«O secret le combat visible dans un vol de feuilles visible dans le gouffre mais non déchiffré.

O noirceur que je donne à mon poing comme une torche.» ${ }^{9}$

Une lumière qui illumine tout l'imaginaire du poète. Le poète retrouve que la source même du génie se retrouve dans un feu inextinguible dispensant une lumière radieuse qui déclenche et nourrit l'inspiration. Observons-le décrire cette source ignée chez le compositeur Monteverdi :

«Monteverdi c'est de la flamme qui se change en ornement sans cesser de brûler.» ${ }^{10}$

Lumière resplendissante qu'irradie une ambiance féerique harmonieuse de "musicalité". Elle s'avère capable de dissiper l'obscurité et ses effets sur l'âme et l'esprit du poète :

«Cela donne plus qu'aucune musique, une idée de feu, de nuits et d'astres.» ${ }^{11}$

L'emblème du génie est toujours le même, qu'il soit d'ordre purement artistique ou littéraire. Jaccottet rajoute le cas du peintre italien Titien et de l'écrivain anglais Shakespeare :

«Cela touche à la fois à Shakespeare et à Titien.» ${ }^{12}$ 
La flamme que diffuse cette lumière est une flamme ascensionnelle comme l'a si bien souligné Bachelard dans son analyse des éléments. Une lumière quasi éblouissante. Sa puissance exceptionnelle est capable de transfigurer l'identité des visages.

Il s'agit toujours du génie de Monteverdi, animé par cette flamme qui :

«S'élevait jusqu'au point extrême du ciel, une puissance transfigurante.» ${ }^{13}$

Le poète fascine par cette lumière, l'appréhende parfois quand il pense à ses origines : l'au-delà ! Affronté à la déchirure suprême, la séparation de l'esprit et du corps après la vie, Jaccottet se méfie de cette lumière qui représente à ses yeux l'au-delà; ce que les êtres humains appellent la mort. Jaccottet essaie par tous les moyens ; notamment celui de l'écriture, d'exorciser cette idée qui le hante.

Hélas ! tout s'avère inefficace, elle est toujours là :

\section{«Il n'est pas un endroit où ne nous débusque le pinceau de lumière mortelle qui est censé nous ouvrir l'avenir.» 14}

Cela incite le poète à mieux saisir le sens de l'éternité indissociable chez Jaccottet de l'amitié. Le sens de cette amitié qu'inspire la forêt en présence des pierres, et tout ce qui compose le sol des sous-bois, permet en effet à Jaccottet de 
mieux comprendre le sens de la bonté et les dimensions de l'éternité qu'engendre la lumière. Apprécions avec le poète cette sensation d'éternité qui l'envahit sous les feuilles des arbres en présence de la lumière :

\section{«Sous ce filtre à lumière, à infini [...] ce qui semble} éternel à force de recommencer toujours le même et sans monotonie.» 15

Cet aperçu sommaire de la nature abstraite insaisissable de la lumière nous incite à aborder l'interférence de la pensée devenant parole sous l'égide de cette lumière bénéfique chez Jaccottet.

\section{Métamorphose de la Pensée en Parole :}

Le monde de la lumière constitue la base même de l'imaginaire de notre poète. Une lumière qui revêt parfois la forme d'un vecteur qui véhicule la pensée. Cette luminosité quoique fugitive, notre intellect s'avère capable de la saisir. Le poète met en évidence cette lumière de l'infini entourant les astres avec son auréole en soulignant son ascendant sur la pensée :-

«Naissance d'un astre. Notre pensée néanmoins saisit cela. Pensée emportée à son tour, paroles éparses, même les plus purs.» ${ }^{16}$

La pensée et la parole représentent un véritable foyer de lumière dans l'imaginaire du poète. Ces pensées formulées 
dans les attrayantes "citations" des écrivains s'avèrent capables d'éveiller et de nourrir l'intelligence du lecteur. La beauté de "quelques" notations de Joubert de Senancour ne manquent pas d'illuminer l'âme et l'esprit du poète : -

«Le rayonnement que prennent certaines notations de Joubert de Senancour.» ${ }^{17}$

Observons de près comment la richesse, l'ampleur et la profondeur de l'imaginaire du poète retrouvera dans la glace à laquelle est souvent associée la lumière, l'émanation des idées, base même de toute parole. L'influence de la glace sur l'imaginaire du poète est quasi surnaturelle. La profonde sensibilité de Jaccottet qui jouit de cette faculté de réunir l'abstrait et le concret, l'invisible et le visible, retrouve dans la glace une source considérable de la pensée et des idées : -

«Glace, lames, feuilles de givre. On voit encore ces choses, et on ne les voit plus comme des choses mais comme émanations, idées, figures, mouvements,

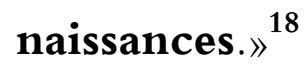

L'association des trois éléments parole - eau - lumière se retrouve parfois dans une image que seules l'ingéniosité et la virtuosité s'avèrent capable d'assembler :

«Des paroles brèves comme une rapide pluie; comme ces lignes qu'elle laisse sur la vitre un instant, brillantes, étoilées.» 19 
Evoquant le côté lumineux, fragile et éthéré de l'inspiration; Jaccottet rajoute à l'élément essentiel de la lumière : l'eau, celui du verre transparent:

«Comment faire sentir, fixer un équilibre fragile, quelque chose d'analogue à une colonne de verre, ou même d'eau s'appuyant sur le vide.» ${ }^{20}$

Cette lumière est aux yeux du poète intermittente; fragile qui peut d'un instant à l'autre disparaître emportant avec elle l'inspiration qui s'étiole progressivement.

Le poète décrit cet état de l'inspiration : un éclair "s'appuyant" sur le vide :

\section{«il brille et $s^{\prime}$ écroule.» ${ }^{21}$}

Décrivant la création littéraire et ses difficultés, le poète évoque toujours la luminosité qui accompagne la parole :

«A chaque instant, c'est comme la première et la dernière parole [...]. Encore une fois l'astre sort du fourreau.» ${ }^{22}$

Cette lumière résiste aux forces sombres de l'obscurité et des ténèbres :

«Encore une fois au soir son bruit contre la mort.» 23 
Cette lumière - verbe et parole émaille les meilleurs écrits du poète qui sont apparentes à des critaux étincelants.

Relevons la confidence de notre poète où se dévoile sa conception de l'écrivain, de tout ouvrage artistique, fondement essentiel du monde culturel constituant une belle symphonie qui englobe la musique, la poésie et la science.

La confidence du poète s'avère d'autant plus révélatrice qu'elle se réalise dans le petit matin avec la première lueur du soleil dans une atmosphère de limpidité :

«Rêve d'écrire un poème qui serait aussi cristallin et aussi vivant qu'une ouvre musicale [...] regret de n'être pas musicien, de n'avoir ni leur science, ni leur liberté.» $^{24}$

Cette lumière qu'irradie la poésie a un effet balsamique sur le cœur. Elle le revigore à l'instar d'une fraîcheur dynamisante :

«[...] La poésie illuminant par instants la vie comme une chute de neige et c'est déjà beaucoup si on a gardé les yeux pour la voir.» 25

\section{C) Son Caractère Concret :}

Le caractère abstrait de la lumière n'exclut guère chez notre poète son impact physique sur lui ; malgré l'éloignement de Jaccottet et la distance qui le sépare des 
astres, il ressent leur parfum grisant. Le pouvoir considérable qu'exerce cette lumière sur le poète est quasi hypnotique. La métaphysique dans cet état second se confond avec le physique. La perception sensorielle se déclenche avec le sens olfactif et visuel qui réagissent les premiers dans cette vision sensationnelle :

«L'astre paraît briller dans une immense fumée, dans un parfum.» 26

\section{Sa Présence Terrestre :}

L'imaginaire du poète imprégné de lumière va jusqu'à la sonder dans la terre. Jaccottet y voit une lumière puissante et potentielle ; celle d'un volcan qui couve :

«Terre comme un feu endormi, allongé sous les arbres.» $^{27}$

Le poète retrouve une sorte d'osmose entre la lumière et la nature. Dans l'imaginaire de Jaccottet cette lumière traverse toute masse élevée de la terre, notamment les dômes et les montagnes.

Le jeu de l'ombre et de la lumière exerce un pouvoir fascinant sur le poète. Observons de près l'effet de cette brillance sur notre poète :

«Vu d'ici, un dôme, un dos d'âne aplati au-dessus duquel le ciel est encore plus lumineux.» ${ }^{28}$ 
Toutes ces masses volumineuses, imprégnés de lumière acquerront les traits spécifiques de cette lumière. Elles deviendront légères, quasi éthérées et diaphanes :

«Sans volume, sans relief, sans détails, s'élevant au-dessus des arbres et là où cela touche leurs cimes, également plus lumineux; d'où l'impression que c'est léger, suspendu ou flottant.» 29

Le poète reconnaît que cet effet insaisissable frise le surnaturel et la magie. La fugacité de la lumière diminue la sensation de lourdeur de toute masse qu'elle traverse :

«Jusqu'ici j'avais deviné un élément essentiel de cette magie : la légéreté.» 30

Effet qui n'en demeure pas moins ressenti physiquement. La masse de la montagne en présence de la lumière devient alors assurance et reconfort :-

«C'était (je crois bien) comme s'il y avait eu à ma gauche une présence $[. .$.$] quelqu'un [. ..] »^{31}$

La paix et la scintillation de la lumière convertit la montagne-obstacle en une masse poreuse ou l'on peut s'abriter et trouver une protection :

«Montagne - maison. ${ }^{32}$ 


\section{La végétation de la lumière :}

Une profonde osmose règne entre le monde végétal et la lumière, une sorte de connexion entre elle et la vie qui fait monter la sève du sol jusqu'aux faîtes-mêmes des arbres à l'instar de la circulation du sang dans l'organisme humain et qui n'est en fin de compte que le flux et le reflux de la vitalité même qui nous habite et qui se reflète sur notre état de santé. C'est à la période de la floraison d'un arbre, la "naissance" de petits fruits aperçus, à travers le bourgeonnement des arbres prodigues et généreux que la lumière scintille annonçant cette aubaine :

«Et puis la première fleur ouverte sous la pluie comme un astre rose. Constellation du pêcher. Elle a la couleur de l'aube. Pêcher, constellation de l'aube.» ${ }^{33}$

Dans l'imaginaire de Jaccottet l'osmose entre la lumière et le végétal est identique à celle qui se reflète entre les astres. Les "constellations" du ciel sont toujours présentes dans l'imaginaire de Jaccottet. La mémorisation de l'univers stellaire est parfaitement identique à celle qui repère la naissance de la lumière dans les bourgeons qui apparaissent et illuminent le jardin du poète. L'interférence entre la lumière céleste et celle de la végétation ne tolère guère l'oublie ni les failles de la mémoire : 
«Contemplateur du zodiaque terrestre d'une galaxie arrêtée dans un jardin. Bientôt ce sera l'acacia, je ne l'ai pas oublié ...» ${ }^{34}$

Le poète est fort convaincu que cette lumière représente la force vitale dans toute plantation. Une lumière à la portée de notre perception visuelle. La sensibilité de Jaccottet perçoit ce "souffle" vital qui circule dans les cellules de l'herbe fraîche qui pousse :

«Les herbes nouvelles d'une luminosité intense, comme éclairées du dedans par de l'or, du jaune d'or.» ${ }^{35}$

La puissance de cette poussée va jusqu'à rapprocher la lumière de la végétation. Soulignons cette similitude :

«Il me semble que la lumière aujourd'hui a grandi comme une plante.» ${ }^{36}$

L'identification va parfois jusqu'à l'association avec l'eau :

\section{«Les feuillages brillants comme de l'eau.» ${ }^{37}$}

La lumière omniprésente dans l'imaginaire de Jaccottet englobe de même les volatiles. Légèreté d'une lumière bénéfique qui rayonne partout annonçant le déclenchement du cycle créateur chez les oiseaux : 
«Cette lumière dans les terres jaunes $[. .$.$] cet œuf$ [...] comme s'il s'y levait un oiseau blanc.» ${ }^{38}$

Cette lumière qui dispense la joie dans tout l'univers invite toute la nature à célébrer les beaux jours verbaux.

La gazouillement des oiseaux qui s'envolent n'est que le signe annonciateur de cette nouvelle saison :

«Oiseaux qui s'envolent des arbres secoués par le vent $[. .$.$] lumière du dedans, monde, bois, éclairés.» { }^{39}$

$\underline{\text { Le caractère aquatique de cette lumière : }}$

Le poète apparente souvent la lumière à l'élément de l'eau : Jaccottet, parfaitement catégorique dans cette association, considère cet élément comme une matérialisation aussi bien qu'une réflexion de la lumière:

«Comme la lune est le miroir du soleil, l'eau est la lumière qui s'enfonce dans la terre, une lumière fraîche, un ciel de septembre. ${ }^{40}$

Le paroxysme de cette comparaison se retrouve dans la lumière étincelante des astres : -

«L'étoile est un feu d'eau, un feu glacé.» ${ }^{41}$

Le raprochement entre les deux éléments est fondée sur la vitalité qui les unit. L'imaginaire du poète y retrouve ses fondements. 
A travers un regard, l'on pourrait mieux observer l'amoindrissement de ce rayon lumineux qui s'étiole dès que l'oeil se ferme :

«Un regard qui se ferme comme s'il y avait moins d'eau dans les vallées. ${ }^{42}$

La lumière des yeux, dans l'imaginaire du poète, reflète somme toute la constitution de la nature de notre masse cellulaire.

«On est fait de lueurs, on n'a plus d'existence qu'un nœud d'eau et de lueurs.» ${ }^{43}$

Quand l'eau se cristallise sur terre, et devient une véritable surface miroitante elle explique davantage le reflet de la lumière des yeux sur le plan planétaire.

Une masse purificatrice qui dissipe le brouillard, annonce le soleil et devient messagère d'une luminosité intense. Les rayons du soleil que reflète la neige, purifient à l'instar de l'eau et débarrassent la nature de tout ombrage et ornement superflu.

Jaccottet souligne l'importance de cette eau glacée et cristallisée, celle de la neige :-

«Glace, limpidité, soleil. De rares nuages, petits accrochés aux montagnes. Tout est purifié, les ornements sont tombés.» ${ }^{44}$ 
Sur la surface enneigée des Préalpes du Sud près de Carpentras, le poète nous confie tout le charme qu'exerce sur son âme la lumière que reflète sur lui le Ventoux :

«Au loin le Ventoux et son dôme brillant de neige. ${ }^{45}$

Cette lumière ne quitte guère la neige lorsqu'elle fond dans les rivières. Au bas de la pente d'une rivière, le poète profite de la beauté du paysage pour nous communiquer la valeur qu'il voue au monde de la lumière qui illumine la surface de l'eau : -

«On voit une couleur d'eau vert clair. Il y a des pierres précieuses de cette couleur là.» 46

Le phénomène de l'eau-lumière se réitère à son niveau microcosmique dans le cas des larmes, qu'il s'agisse de joie ou de chagrin, les larmes nous permettent d'avancer dans la vie. La clarté qui scintille dans les larmes détruit les obstacles, effrite les écueils à l'instar de l'eau qui dissout la matière solide pour nous frayer des chemins et nous ouvrir des horizons.

Soulignons ce panégyrique de la clarté, perçue par le poète dans les pleurs-mêmes qui inondent nos paupières : -

«Y aura-t-il des larmes assez claires pour nous creuser un chemin dans ces terres.» ${ }^{47}$ 
L'eau-lumière a toujours hanté l'imaginaire du poète: la translucidité qui caractérise la lumière se reflète dans toute coulée légère. Pour mieux fixer l'instant de passage de cette lumiere, Jaccottet la retrouve sur la surface transparente des vitres quand les gouttelettes de pluie s'y agglutinent. Une goutte de pluie sur une vitre, représente dans l'imaginaire du poète "l'arrêt" du temps fixé sur un rayon de lumière. La vitre transparente capte et fixe la lumière fugace, l'espace de quelques secondes pour refaire l'éternité dans l'âme et l'esprit du poète. Observons de près cette magie de la lumière chez Jaccottet à travers une goutte de pluie "attachée" à la vitre : -

«Chaque goutte de pluie, plus claire, plus brillante où elle est la plus lourde, attachée à la vitre par son ombre.» 48

Le charme et la saveur de ces instants ne sont pas loin de ceux qu'éveille la dégustation d'un fruit frais.

\section{«Un très petit fruit limpide.» 49}

Toute cette saveur gustative matérialise l'effet ressenti lors de la scintillation des gouttelettes de pluie :

«Il n'y a que les eaux qui tombent du ciel qui puissent subsister en gouttes et brilles comme les rosées.» 50

Cet élément aquatique qui véhicule et reflète la lumière, peut le cas échéant, lorsqu'il s'accumule devenir un obstacle 
devant la translucidité de cette lumière. Elle devient alors obstacle auquel se heurte et s'immobilise la lumière.

Suivons de près, cette situation où la densité de l'eau devient voile, voire rideau opiniâtre devant la libre circulation de la lumière. Il s'agit en l'occurrence du cas de la pluie quasi torrentielle : -

«Les mobiles, les translucides constellations de la pluie sur les vitres qui ne sont au loin que voiles en marche, rideaux qui se ferment. ${ }^{51}$

\section{II- L'importance de cette lumière :}

\section{A) Source de vie et Animation :}

La lumière dans l'oeuvre de Jaccottet rime toujours avec vie, euphorie et entrain. La nature représente le meilleur support matériel qui nous communique les sensations du poète. Le poète s'avère un "fin limier" qui parvient aisément à capter le rayonnement secret et discret de la terre quand elle est bien imprégnée de vie, et particulièrement de la rosée du matin.

Une sorte de rayonnement qui se diffuse en douceur et en sérénité. Des rayons qui traversent les pétales couleur de rose :

«Ces derniers pétales de roses, couleur [...] de feu secret, ces aveux de la terre.» ${ }^{52}$ 
La consomption même de cette scintillation se réalise en douceur et sérénité :

«Tandis que le jardin se consume sans flamme...se dessèche.» ${ }^{53}$

La lumière de ce feu doux et discret monte jusqu'aux cîmes des arbres rivalisant avec la beauté même de l'or :

«Ce n'est pas de l'or sur les arbres, les vignes, c'est plutôt une couleur de flamme très clair...et encore donne-t-elle un idée de repos, pas de chaleur.» ${ }^{54}$

Ce qui met en évidence l'euphorie déclenchée par la lumière lors du printemps et de la floraison des arbres, c'est son caractère incoercible qui contraste avec l'amertume qui gagne le poète pendant l'automne. L'écart est vertigineux entre les deux saisons. Observons de près l'effet ressenti lors de la disparition de la lumière pour mieux saisir le culte du poète qui lui est voué.

«Fête, fatale, funeste en un sens.» ${ }^{55}$

Ce sont les couleurs étiolées qui remplacent la splendeur et l'épanouissement du printemps. Avec la disparition de la lumière, l'assurance l'abandonne :

«Tout ce qui est sûr s'éteint comme une flamme qui s'éteint. ${ }^{56}$

Et plus loin : 
«Lampe éteinte - tombeau de la pluie. Lampe soudain éteinte. ${ }^{57}$

En hiver l'éclat de la lumière qu'irradie la neige donne une forte impression d'animation à la nature en dépit de l'immobilité apparente de ce "bloc" de glace qui couvre la végétation et les masses rocheuses. Tout ce qui est "immobile" et "inébranlable" à la surface de la terre n'est qu'apparence, car la lumière est toujours là avec son animation et sa vitalité :

«Il faut dire ici un grand espace d'air de mobilité, d'éclat, d'animation au-dessus de l'inébranlable. ${ }^{58}$

Plus loin, le poète nous explique l'effet de cette lumière sur la matière apparemment inerte. Une sensation de légéreté vivifiante, une force tranquille ressortent du végétal. Le "dynamisme" et l'activité qui grouillent dans le sol s'avèrent toutefois impressionnante par leur caractère sérieux, sévère, sans fanfaronnade ni orgueil :

«Mais la roche n'est pas lourde, elle est grave, sévère, elle fait penser à des guerrier invincibles mais sans jactance ni panache, à une force vraie, silencieux.» 59

Dans l'imaginaire du poète, cette force de vie qui se dégage de la lumière ne connaît que la productivité efficace, humble et modeste. Elle ignore, comme il le confirme, l'orgueil. Ne perdons jamais de vue que notre poète est un 
inconditionnel adepte de l'effacement. La lumière douce qu'irise la floraison de l'amandier et du pêcher, n'a pas le même "vernis" des cerisiers et des pruniers : -

«Floraison de l'amandier, du pêcher comparée à celle des cerisiers, pommiers, pruniers, orgueil du Nord.» 60

Le charme particulier qu'exerce le soleil sur l'imaginaire du poète s'explique par son rôle vital dans l'animation de l'univers.

Jaccottet est toujours à l'affût de cette panacée qui réchauffe la planète. Soulignons la comparaison établie par le poète entre la lumière artificielle et celle du soleil pour mieux appréhender l'importance qu'il voue à cette dernière. Le brouillard qui couvre la nature permet de mettre en évidence la prééminence de la lumière naturelle :

«Ici une lampse allumée dans la rue, jaune comme un soleil sans rayon.» ${ }^{61}$

Le reflet de cette lumière acquiert toute sa valeur de l'or auquel elle est comparée :

\section{«Là, une porte dorée. ${ }^{62}$}

La lumière devient projecteur sur le monde extérieur.

Une sorte de réverbère dont la puissance de projection est teinte d'une subtilité extrême à l'instar d'une ombre qui 
guette le premier faisceau de lumière solaire qui illuminera le monde pendant la journée.

\section{«Une ombre regardant le temps qu'il va faire sur le jardin.» 63}

La vitalité et l'importance de cette lumière s'explique par sa quasi omniprésence dans chaque élément qui anime la vie.

Elle est l'origine même de la vie sur terre. Une importance d'autant plus cruciale qu'elle participe de l'invisible ; source de son rayonnement. Le poète va directement au vif du sujet. Décrivant l'invisible ,il nous confie :

\section{«'ai vu en lui la source du jour.» ${ }^{64}$}

Se trouvant dans une situation embarrassante, Jaccottet rédige un poème "Approche encore" qui lui a été dicté dans un de ces moments où il avait perdu "la paix de l'âme". Notre poète reconnaît toutefois son erreur et sa fébrilité devant la puissance de cette source du jour. Entendons le dire dans ce poème avec une certaine perplexité :

\section{«Sa revanche est d'apparaître innommable sous} cette clarté.» ${ }^{65}$

La lumière de l'inspiration qui déclenche la verve poétique s'avère particulièrement importante pour maintenir 
et nourrir l'intuition du poète. Jaccottet décrit l'effet bénéfique de la lumière de l'intuition qui inspire et instruit : -

«J'eus alors l'impression que m'était dévoilé l'ordre du monde.» ${ }^{66}$

Une lumière qui rassure en dissipant le voile des ténèbres. Jaccottet continue :

«Le voile des ténèbres, de l'obscurité disparaît [...] le voile qui nous sépare d'ordinaire du réel se déchira.» ${ }^{67}$

Dans la lumière tout le monde de l'art et de la peinture se module pour exprimer la beauté de cette animation.

La lumière à l'effet et le savoir faire d'un peintre. Elle engendre art, doigté et génie. Son emprise sur l'âme et l'esprit relève de l'ingéniosité même de Van Gogh fignolant un tableau :

\section{«Quelques fermes peintes par la lumière.» ${ }^{68}$}

Lorsque la neige, cette eau gelée, à laquelle est assimilée la lumière communique son dynamisme de joie et de beauté au monde végétal qu'elle imprègne ; la couleur grise des troncs s'atténue pour céder à la beauté du rose sous l'effet de la lumière.

«Troncs lisses, d'un gris à peine jaune ou rose par la faible lumière.» 69 
La forme même élancée et gracieuse de ces troncs, s'explique par l'état d'âme du poète qui fait le panégyrique de la lumière dans une ambiance féerique. Une lumière toujours accompagnée de beauté immatérielle qui entraine l'esprit dans son ascension vers les cîmes de la spiritualité au "rythme" ascensionnel d'une ramure pure qui ignore la tortuosité et les nodosités de la végétation qui poussent sur les rochers.

Suivons de près cet état d'âme qui gagne le poète dans cette ambiance sereine et pure de lumière dans un mouvement ascensionnel ayant pour support physique la ramure des arbres :

«Leur ramure ascendante et simple au contraire des arbres tordus, noueux qui

\section{habitent le rochers.» ${ }^{70}$}

La beauté de la vie et de l'animation qui imprègnent le paysage dispense une sensation de légèreté. Jaccottet en osmose avec l'euphorie de la végétation n'y voit que légèreté et ascension.

«Un lieu de frêle ascension [...] si différent des groupes de cercles augureux des chênes couverts de lierre, avec leur force noire, leurs chaînes. ${ }^{71}$

La lumière de l'inspiration qui déclenche la verve poétique s'avère particulièrement importante pour maintenir 
et nourrir l'intuition du poète. Jaccottet décrit l'effet bénéfique de la lumière de l'intuition qui inspire et instruit : -

«J'eus alors l'impression que m'était dévoilé l'ordre du monde.» ${ }^{72}$

Une lumière qui rassure en dissipant le voile des ténèbres. Jaccottet continue :

«Le voile des ténèbres, de l'obscurité disparaît [...] le voile qui nous sépare d'ordinaire du réel se déchira.» ${ }^{73}$

Dans la lumière tout le monde de l'art et de la peinture se module pour exprimer la beauté de cette animation.

La lumière à l'effet et le savoir faire d'un peintre. Elle engendre art, doigté et génie. Son emprise sur l'âme et l'esprit relève de l'ingéniosité même de Van Gogh fignolant un tableau :

\section{«Quelques fermes peintes par lumière.» ${ }^{74}$}

Lorsque la neige, cette eau gelée, à laquelle est assimilée la lumière communique son dynamisme de joie et de beauté au monde végétal qu'elle imprègne ; la couleur grise des troncs s'atténue pour céder à la beauté du rose sous l'effet de la lumière.

«Troncs lisses, d'un gris à peine jaune ou rose par la faible lumière.» ${ }^{75}$ 
La forme même élancée et gracieuse de ces troncs, s'explique par l'état d'âme du poète qui fait le panégyrique de la lumière dans une ambiance féerique. Une lumière toujours accompagnée de beauté immatérielle qui entraîne l'esprit dans son ascension vers les cîmes de la spiritualité au "rythme" ascensionnel d'une ramure pure qui ignore la tortuosité et les nodosités de la végétation qui poussent sur les rochers.

Suivons de près cet état d'âme qui gagne le poète dans cette ambiance sereine et pure de lumière dans un mouvement ascensionnel ayant pour support physique la ramure des arbres :

«Leur ramure ascendante et simple au contraire des arbres tordus, noueux qui habitent les rochers. ${ }^{76}$

La beauté de la vie et de l'animation qui imprègnent le paysage dispense une sensation de legerete. Jaccottet en osmose avec l'euphorie de la végétation n'y voit que légèreté et ascension.

«Un lieu de frêle ascension [...] si différent des groupes de cercles augureux des chênes couverts de lierre, avec leur force noire, leurs chaînes. ${ }^{77}$

B) Source de Joie et de Bonheur : 
Le poète apprécie l'effet bénéfique de la lumière sur l'âme et l'esprit. Jaccottet y retrouve une source de douceur et de paix de l'âme et ne tarde guère à y recourir :

«Une douceur pareille à celle de la lumière quand le soleil descendant vers l'ouest, la forêt plus longue et plus dorée.» ${ }^{78}$

La lumière annonciatrice de bonheur et de joie nous est communiqué par tous les éléments de la nature notamment les cristaux de neige. Cette joie se laisse facilement percevoir quand la neige qui charrie la lumière n'a pas encore disparu à la surface du sol. Sa disparition n'est que regret et obscurité :

«La neige [...] s'étant éteinte [...] tout s'est assombri.» ${ }^{79}$

Le regret lancinant du poète est souvent ressenti lors de sa privation de cette lumière ; visuelle aussi bien qu'émotionnelle. Le beau tableau que dessinait la neige disparaît en emportant la gaîté qu'il prodiguait : -

«Cette si légère charge sur les arbres, c'est comme si nous regardions à travers un tulle [...] Une gaieté enfantine gagnait tout le village.» ${ }^{80}$

La jovialité de la lumière se communique à tout ce qu'elle imprègne. Elle s'avère capable de convertir en rose la blancheur même des murs d'une chambre qu'elle gagne : 
«Intimité de la lumière dans la chambre sur le papier blanc qui à son tour est devenu presque rose. ${ }^{81}$

La fumée des cheminées nourries du feu des bois a toujours été le symbole de la joie. Il en est de même chez Jaccottet un véritable augure de joie et de gaieté.

Elle s'accompagne souvent du signe même de la joie : la couleur rose. Le poète compare les arbres défeuillés du bois de "Lez" avec leurs troncs minces et droits à la fumée. La lumière qu'elles irradient nous communique cette impressionnante de fluidité :

Nous nous sentons plus légers :-

«Quelque chose de léger qui monte et se ramifie, de clair, presque rose.» ${ }^{82}$

La lumière convertit tout ce qu'elle imprègne en rose. La neige souvent assimilée à la lumière ne se contente pas de mettre en relief la beauté de la nature mais l'entoure d'une roseur fascinante : -

«Le bois ne se distingue de la terre que par sa forme. Tout est couleur de terre, presque couleur de rose, jusque là où campe la neige.${ }^{83}$

L'emphase et la verve poétique n'excluent pas chez le poète, la lucidité : Jaccottet est bien conscient que toute cette beauté participe de l'immatériel, de l'éthéré et de l'invisible. 
Elle ignore la concupiscence de la chair et des plaisirs sensuels qu'inspire souvent la vie en rose : -

«Que dire de ce blanc et de ce rose ? La rose de la carnation est d'un autre ordre et il y a autour du mot rose beaucoup d'impressions qu'il faudrait effacer, en particulier les érotiques. ${ }^{84}$

Jaccottet continue d'évoquer cette ambiance -d'ailleurs comme nous l'avons souligné assimilées à la lumière ; nourrit les arbres. Cela n'est pas sans nous rapprocher des idées chères à Jean-Jacques Rouseau, celle de l'influence éducative et régénératrice de la nature sur l'homme. Sur un sol couvert de neige et irradiant de lumière Jaccottet nous invite à profiter de ces moments d'ataraxie : -

«Car il s'agit de la chose la plus pure. Ainsi même les blessures n'ont-elles pas l'aspect répugnant, effrayant des blessures des bêtes et des hommes. [...]. Ni le bois ni la feuille morte n'effraient, ne repoussent. Horreur du sang [...]. La sève qui coule n'est guère qu'une larme.» ${ }^{85}$

Souvenons-nous que les larmes, comme nous l'avons déjà mentionné, sont associées à la lumière.

Jaccottet reconnaît la puissance de la lumière et l'impact de sa galvanisation sur notre système sensitif. Ecoutons-le chanter symphonie mélodieuse euphorisante où la lumière 
revogore les sens et imprègne l'âme de joie. Plein de confiance en son effet salvateur, il ira jusqu'à dégager davantage de rayonnement dans la couleur bleuâtre du soir que dans la lumière du jour. Le poète nous invite à partager son émoi dans un panorama séraphique :

«Au-dessus, de ces parfums, de ces émanations, de cet émoi; de cette activité, le bleu surprenant du ciel [...] profondément bleu, beaucoup plus bleu que de jour, et les nuages aux dômes éblouissants.» 86

La lumière n'engendre que la légèreté du monde immatériel. Dans une belle image qui englobe les éléments auxquels sont associés la lumière, l'eau et le feu ; Joccottet nous décrit l'effet balsamique de cette atmosphère qui ignore les complications de la vie terrestre figée. La fluidité de l'eau charrie toutes les difficultés et ne laisse percevoir qu'un panorama vaporeux de transparence et de sérénité :

«Tandis que le long de la rivière tout est passage : eaux, fumées, allée sous l'arceau léger des vapeur.» ${ }^{87}$

Jaccottet décrit la légèreté de cette vie édénenique et fait la part des choses entre les deux mondes qui fondent notre existence, la vie terrestre avec ses "nodosités" et son opacité et celle de l'éternité faite de transparence enrobée de lueur et d'éclat : 
«Comme deux vies, deux pensées possibles, l'une noueuse, attentive, méditative et liée à la nuit, à la pierre druidique, penchée sur la bouche du sol ; l'autre vive légère [...] au fil étincelant des jours.» ${ }^{88}$

La lumière ne peut refléter que la sérénité et l'ataraxie de l'âme.

Le poète insiste sur l'efficacité de la lumière contre les pièges de la morosité et l'égarement de l'âme. Son infaillibilité positive s'avère capable de sauver une âme du néant. Cette lumière représente l'ultime chance et le dernier recours contre l'écroulement. Jaccottet nous exhorte à bien apprendre l'art et la manière de combattre ce fléau qu'est la morosité :

«Que la tristesse taciturne couve au moins cette extrême chance de la lumière.» ${ }^{89}$

Un véritable rempart contre les surprises et les caprices du désarroi autant que les fleurs le sont contre la misère.

«Que cette extrêmité de la misère maintienne la chance des fleurs. ${ }^{90}$

La beauté d'un jardin enveloppé de lumière exorcise le découragement et dissipe l'effet pernicieux de la tristesse.

Dans les jardins et les prairies grisées de lumière ; la joie chante à l'unisson avec la nature. Le poète nous invite à 
suivre sa méthode pour exorciser les maux de l'âme et de l'esprit et ce grâce à la brillance de la nature.

«Il y a quelque chose à dire sur cette impression de brillant, de légèreté de transparence sur l'envie de marcher dans ces jardins, ces prairies, au pieds de ces montagnes, sur cette impression irrésistiblement paradisiaque. On ne saurait préciser pourquoi.» ${ }^{91}$

Les animaux partagent la même joie et les mêmes sensations de l'homme. Dans une scène non dénuée d'une certaine magie poétique, le poète évoque l'effet bénéfique de la lumière lunaire qui rassure et rassérène un troupeau de moutons. Il met en évidence la reconnaissance de ce troupeau qui adresse ses remerciements à cette lumière généreuse.

Scène pathétique où se mêlent le sacré et le quotidien à la fin d'une journée qui nous rappelle le soir de la vie :

«Transhumance [...] lune croissante, bêlements accordés à sa lumière. Âmes attachées au mur. Béliers leurs combats, les bêtes forment cercles, scène sacrée. Le soir tombe. Poussière.» ${ }^{92}$

\section{C) Source d'espoir et d'entousiasme :}

Toute lumière si ténue soit-elle, puise ses ressources dans la force invincible de l'invisible. La clarté de cette lumière a toute la puissance d'une aile qui nous arrache à la 
perdition et au néant. Jaccottet associe la clarté, véritable rempart contre la voracité de l'abîme, à une flèche qui nous donne des "ailes".

«A peine une aile, à peine une flèche un peu claire. ${ }^{93}$

Dans l'abîme même Jaccottet puise la lumière pour vaincre les forces du mal, de la défaite. La clarté rime toujours avec espoir et espérance. A l'instar d'une voix claire et puissante, la défaite changera de ton en presence de la lumière.

\section{«Que notre défaite rayonne et claironne.» ${ }^{94}$}

L'espoir qu'insuffle la lumière à toute la nature représente une source inestimable de chaleur et de joie. C'est une "arme" contre le grelottement de la nuit et le poids de la glace difficilement supportés par le feuillage des arbres "en loques". Soulignons cette impatience de la nature en attendant le jour :

«Avant le jour, l'hiver, entre montagnes et nuées sombres, annonçant l'armée lumière.» ${ }^{95}$

Le poète connaît bien les avantages de la lumière et son effet bénéfique sur l'âme et l'esprit. Le poète retrouve en elle une source de douceur et de paix de l'âme et ne tarde guère à y recourir : 
«Une douceur pareille à celle de la lumière, quand le soleil descendant vers l'ouest, le fait plus longue et plus dorée. ${ }^{96}$

Pour mieux comprendre l'effet bénéfique, salvateur et réconfortant de la douceur de cette lumière, suivons de près cette épreuve de fragilité morale du poète en absence de la lumière et nous mesurons l'ampleur de sa valeur inestimable :

«Le recommence à marmonner contre la mort. En m'effondrant je persévère. ${ }^{97}$

Ce n'est que le jaillissement de la lumière dans son esprit qui dissipera le doute et renouvelles le moral du poète :

«Je vois l'effondrement qui brille, et toute la distance de la terre illuminée. ${ }^{98}$

Et comme par un coup de baguette magique, sa torpeur et son angoisse disparaîtront et cèderont à cette douceur salvatrice qui a toute la puissance des ailes qui arrachent un naufragé des abîmes du doute et de la perdition. Jaccottet nous invite à partager ces moments d'apaisement :-

«Une douceur insoutenable, une aile sous le couvert sombre des nuées.» ${ }^{99}$

Jaccottet a appris à apprivoiser la lumière et nous exhorte à suivre sa méthode. Derrière chaque nuage veille une lumière ; il suffit d'y croire pour que cette obscurité se 
convertisse en lumière et les moments de perdition deviennent assurance victorieuse. Cette leçon de sagesse nous apprend que vouloir c'est pouvoir et que l'impossible n'est jamais invincible. Il suffit d'essayer, de "voir" : -

«L'ombre m'ouvre les yeux et le rapprochement de l'impossible au fond du jour, l'invasion au fond de moi victorieuse.» 100

La lumière dans l'imaginaire de Jaccottet a toute la puissance d'un "soc" métallique. Rien ne lui résiste.

Le flamboiement de la lumière vaincra toujours les "forces" traitres de l'effondrement :

«A bout de force dans cette aube du novembre [...] Je vois le soc du froid qui s'avance et flamboie.» ${ }^{101}$

Et l'ombre du toute cèdera et deviendra le complice même de la lumière.

Dans une image fort significative ; nous assistons à l'oeuvre du duo "ombre-lumière" apparemment inconciliable : «Et en arrière dans une lumière accrue l'ombre laboure.» 102 


\section{III- ÉNERGIE de cette LUMIÈRE :}

\section{A) Son énergie ignée :}

La lumière dans sa forme intensive peut devenir une source d'énergie. Jaccottet souligne le dynamisme de la lumière qu'irradient les flammes et met en évidence la chaleur qu'elles dispensent :

«Une chaleur elle aussi pleine de force et de constance, mais éventée avec tranquillite par la mer.» ${ }^{103}$

\section{- Un feu de Braise :}

Cette lumière qui anime la vie sur terre, n'est en fin de compte que les rayons qui diffusent la chaleur d'un feu ardent et dont les nuances varient d'un être à l'autre. Des êtres doux, d'autres "bouillonnants" chez qui l'intensité de la vie se mesure en fonction de l'ardeur de cette "flamme-vie". Les poètes en général, grâce à leur sensibilité intuitive, et Jaccottet en particulier, de par la pronfondeur de cette sensibilité qui le caractérise, sont les premiers à vouer une importance considérable à cette notion. Elle est sans équivoque chez notre poète :

«Toute chose visible comme des cris ou des soupirs de l'invisible, souffrant d'être invisible, comme des espèces de flammèhes ! ${ }^{104}$ 
Cette force de la vie anime l'univers, se manifeste dans l'imaginaire de Jaccottet avec toute l'énergie capable de le galvaniser. Dans son activité paroxystique elle a toute la puissance de la foudre :

«Parfois, il nous semble que les soudaines ouvertures se creuse, devant nous dont la direction désigne un centre, comme produit par la foudre.» ${ }^{105}$

Une énergie qui a toute la tonicité du flux et du reflux du sang dans un organisme vivant ou de la sève qui maintient la vie dans la végétation.

A l'instar des pulsations vitales qui charrient la vie dans nos veines, cette énergie "grondante" se radoucit dans son accalmie, dans nos moments d'inactivité de la détente :-

«Et si quelque chose alors en nous se répercute et gronde comme un ébranlement prometteur déjà c'est un autre jour, une autre nuit, et tout pourrait aussi bien s'achever avant que rien ne se fut produit.» ${ }^{106}$

Cette lumière qui représente pour Jaccottet toute l'énergie et le dynamisme qui l'animent, le protègent, le guident et le rassurent est une véritable panacée qui nourrit son espoir sur terre et au-delà de cette vie terrestre. Soulignons cette assurance qui l'imprègne quand il chantonne la lumière irradiée par ce feu de braise tonique et revitalisant 
qui l'entoure, ne l'abandonne jamais au delà de la vie sur terre

«J'avais un miroir, plus, ou ce n'était pas moi que je voyais. Mais des paupières fermées sur des braises.» 107

Le poète se voit "traversait" le miroir et quittant la vie sur terre, départ non dénué de joie, d'apaisement trahi par la couleur rose :

\section{«Un monde rose et profond.» ${ }^{108}$}

Les poètes, mieux placés pour appréhender l'énergie qui diffuse cette lumière ; perçoivent un feu dont la puissance commence par la douceur même, celle de la vie qui anime l'organisme humain mais qui peut atteindre une chaleur de braise chez quelques personnes qui jouissent d'une énergie exceptionnelle et qui se reflète dans le regard même de chaque être humain. Jaccottet avec sa grande sensibilité de poète mesure ces nuances de la lumière dans l'univers. Il connaît bien que la terre que la terre dans sa gravitation cosmique en tournant et s'approchant du soleil, la lumière douce de l'aube peut acquérir la chaleur nécessaire pour chauffer notre planète. Le poète décrit cette notion scientifique élémentaire d'une manière poétique : -

«Oh, ce feu qui court encore une fois à l'aurore.» 109 


\section{- Le Feu de l'Amour:}

La lumière dispense généreusement une énergie de feu dont la chaleur est associée à l'Amour dans l'imaginaire du poète. Toute la nature partage les convictions de Jaccottet. A commencer par les montagnes :

«Le feu qui s'embrase parce que les montagnes sont couchées [...]. Montagnes rêvant amoureuse.» ${ }^{110}$

Cette conception de l'Amour et de son énergie associée au feu constitue une partie intégrante de l'imaginaire du poète.

Un feu qui jaillit des abysses-mêmes de notre planète pour inculquer la vie à la sève qui monte dans la végétation grâce à la théorie de la combustion qui régit toute sa vie, sur terre. Le meilleur symbole de l'amour, la fleur nous élucide parfaitement.

Ce cycle de l'énergie vitale qui couve dans les souches souterraines de notre globe terrestre. Suivons de près cette éloquente équation où se conjuguent l'abstrait et le concret pour nous donner une fleur :

«Fleurs : Elles se préparent au milieu des ossements, dans la maison souterraine chez les morts, ou aussi dans la terre des rêves, dans une masse épaisse au centre de laquelle il y a un feu.» ${ }^{111}$ 
Cette force d'Amour n'est en fin de compte que celle qui anime la vie sur terre. Décrivant la floraison des premières giroflées avec l'inflorescence des petites fleurs, Jaccottet met en relief cette puissance tranquille de la lumière, qui insuffle la vie à l'inflorescence des bourgeons :-

«Comme s'ouvrent les chatons, cette fourrure d'où surgit silencieusement un feu d'artifice durable, presque une nébuleuse ou de la poudre. Ce qui se passe sans aucun bruit perceptible,voilà encore un sujet d'étonnement.» ${ }^{112}$

\section{B) Son énergie solaire :}

L'énergie de cette lumière qui exerce sur le poète un pouvoir quasi surnaturel, représente la source même de son imaginaire et de son inspiration ainsi que celle de la vie sur terre.

Jaccottet s'oriente souvent vers le soleil, cette massive étoile sphérique autour de laquelle gravite la terre pour élucider et comprendre l'origine de l'énergie et de la chaleur qui entretiennent et maintiennent la vie et dispensent la lumière sur terre.

Dans une citation de Parménide traduit par Ives Battistini, Jaccottet met en évidence le sens de l'Eternité que symbilisent les rayons du soleil. 
En dépit de l'absence provisoire de ses rayons pendant la nuit, l'imaginaire de Jaccottet est quasi hypnotisé par la fascination qu'exerce sur lui la lumière:-

«Une lumière empruntée rôde pendant la nuit autour de la terre $[\ldots]{ }^{113}$

Elle symbolise aussi bien la force vitale suprême que la matérialisation visuelle de l'Eternité :

«Eternellement tournée vers les rayons éclatants du soleil.» ${ }^{114}$

Cette lumière dispensée et irradiée par le soleil, emblème de la vie sur terre, représente une source prodigieuse et généreuse de chaleur. Le feuillage des arbres essaie de la radoucir quand sa chaleur excède les normes de notre besoin quotidien.

Le poète nous communique ce merveilleux échange entre le langage de la végétation et la prodigalité du foyer de l'univers :

《[...] entre dans le cercle des chênes [...] chaînes ornées de lierre $[\ldots]$ et la lumière est tempérée par leurs feuilles.» 115

Cette vitalité est d'autant plus considérable qu'elle est suivie par une phase fatidique ; celle de notre disparition quand la lumière s'étiole et que tout craque : 
«Puis toutes choses explosent, non seulement le lieu est emporté, mais son souvenir et la louange du lieu [...] le sol, la table se brise.» ${ }^{116}$

Pour mieux saisir cette fascinante équation entre la lumière-vie et l'obscurité il serait tentant d'accompagner le poète dans ses pérégrinations méditatives sur ces deux notions.

Alors que la lumière, génératrice de vie et d'animation, rime avec mouvement ; l'obscurité, reflet de la mort n'est qu'immobilité. Soulignons cette image frappante chez Jaccottet qui évoque la tombe et ce qui l'accompagne de terre et d'obscur.

«Dire aussi la fleur qui se pose sur les bois, sur la poussière, et la roche enguirlandée de lierre, monument ou tombe immémoriale.» ${ }^{117}$

Jaccottet associe l'obscurité directement à tout ce qui évoque la mort avec son cortège funèbre en l'absence de la lumière. Il continue :-

«Choses vieilles, massives, fortes, sombres, tenaces [...] silencieuses.» ${ }^{118}$

Plus loin, le poète insiste sur la tenacité du lierre en absence de la lumière. Le lierre qui doit un jour "orner" notre tombe quand le dynamisme cessera de nous animer: - 
«Immémoriale tombe ou monument sans ornement que de lierre tenace.» ${ }^{119}$

Sous l'emblême de l'obscurité, c'est l'image des funérailles qui hante l'imaginaire du poète avec son décor funèbre : -

«Une guirlande au promeneur disant bronze de guerre ou verdure nocturne.» ${ }^{120}$

Alors que l'existence sur terre, résumée dans ses derniers moments, le soir de la vie épouse l'ombre et l'obscurité, la lumière de l'éternité demeure intacte. Lumière apparentée à celle qu'irradie le Ventoux.

«La grande aile de l'ombre le soir, sa marche sur

les champs, les jardins, tandis qu'au loin le Ventoux brille encore intact.» ${ }^{121}$

Jaccottet le confirme plus loin, sous le signe des souvenirs qui ont truffé notre vie et qui finissent accumulés dans l'herbe qui entoure "l'immémoriale tombe" :

«A mesure que le bras, le fond est plus sombre, plus vert, plus poignant de je ne sais quel signe d'enfance, de souvenir de temps accumulé dans l'herbe.» ${ }^{122}$ 
L'imaginaire du poète galvanisé par le dynamisme de la lumière, y voit tout le sublime de l'éternité dans un panorama qui enveloppe les hauteurs :

«Le haut et le lointain devient plus rose, plus doré, plus lumineux.» ${ }^{123}$

Mieux encore :

«Dans le ciel se sont les fleurs, des lambeaux de feu, de la lumière modelée en nuages.» ${ }^{124}$

\section{C- Son dynamisme :-}

Le dynamisme de la lumière se concrétise dans la célérité de son mouvement. L'énergie de cette lumière se mesure avec la vitesse du vent qui secoue les nuages. Cette secousse émotionnelle n'est pas exempte d'un plaisir visuel suscité par la couleur même de cette lumiêre :

«Lumiere dorée dans l'air froid. Comme elle quitte vite les arbres et s'élève jusqu'aux nuages emportés par le vent.» ${ }^{125}$

Dans l'espace et sur terre cette lumière vivace opère. Le poète retrouve dans la couleur verte et "claire" d'une rivière une ressemblance avec les "pierres précieuses".

Dans l'imaginaire du poète toute la valeur de cette couleur d'une lumière s'explique par l'énergie de son 
mouvement qui se repère par le déplacement des lines d'écume "vive" à la surface de l'eau :

\section{«Une ou deux lignes d'écume vive en travers pour signifier son mouvement rapide comme sur place.» ${ }^{126}$}

Cette lumière qui représente la puissance dynamique du mouvement dans l'espace et sur terre ; animant toute les créatures y compris la végétation n'en demeure pas moins dans son dynamisme régénérateur et vivifiant, le symbole de la fugacité. Fugacité de l'éphémère de la vie tout court. Evoquant le poète Bacho, Jaccottet met en évidence ce trait caractéristique de la lumière perçu à travers l'éclair :-

\section{«- Combien digne d'admiration}

- Qui ne pense point, la vie est fuyante.

\section{- En voyant l'éclair.» ${ }^{127}$}

Sa fugacité ainsi que son mouvement sont en fin de compte une preuve quasi irréfutable et irrécusable de son activité universelle. Ne se reflète-t-elle pas sur notre état moral! Notre bien-être physique et intellectuel.

La médecine contemporaine ne prône-t-elle pas le recours à la lumière pour stimuler le moral de l'être humain et galvaniser ses ressources biologiques et secouer ce qui stagne en lui de vitalité inexploitée ! 
Le poète applique en effet cette théorie et en ressent l'effet concret et bénéfique :-

«Le vol de la lumière, cela seul m'éveille encore. Elle chasse le sommeil, ce lointain sommeil.» ${ }^{128}$

C'est là que réside son pouvoir régénérateur. L'effet revigorant de la lumière renouvelle et secoue l'énergie et le dynamisme de notre organisme. Sur le plan exclusivement médical, il est prouvé comme nous l'avons mentionné, que le moral de l'homme change considérablement et diminue en absence de la lumière. Le rentabilisation qui nous gagne avec son apparition est observée par le poète : -

«S'efface, apparaître. Ainsi ces jeux de lumière. On se réveille d'une longue torpeur [...].» ${ }^{129}$

Le retentissement de cet effet se reflète sur la nature apparemment inerte.

\section{«Epanouissement bref contre les rochers.» ${ }^{130}$}

Le dynamisme ultra-puissant de cette lumière n'est point exempt de danger. Une lumière excessive peut acquérir une puissance telle qu'elle exige un rempart et une protection. L'image la plus approximative de l'imaginaire de Jaccottet démontrent l'ampleur de ce revers de la médaille est celle d'une quelconque bâtisse contre un soleil de plomb : 
«La maison ici en cette saison n'est qu'une réserve d'ombre, une petite forteresse contre les armées solaires.» 131

La maison s'avère capable de convertir cette "masse de plomb" en fraîcheur. Elle acquiert de la sorte tout le charme et la beauté d'un château. Une véritable "réserve" de douceur

«Un château d'eau, un château de fraîcheur.»» ${ }^{132}$

Jaccottet tient à préciser ce détail. Il nous rapproche son image en faisant le parallèle entre la mer et la terre ; le mouvement de la lumière et le statisme placide du sol : -

«Entre l'éclat mobile de la mer et le calme fécond des sols.» 133

Le dynamisme, parfois agressif, d'une lumière puissant s'apparente au heurt violent d'une masse "métallique" dont le retentissement se laisse facillement percevoir par l'impact de sa violence.

«Choc d'une puissance acharnée contre une puissance tout immobile.» ${ }^{134}$

Cette surprenante agressivité accentue dans l'imaginaire du poète le retentissement de cet entrechoquement. 
Et le simple éclat devient un impressionnant assourdissement ; Jaccottet décrit la violence franchie par la lumière :

«A cette lumière limite se produit un feu blanc, une déflagration de gouttes d'eau.» ${ }^{135}$

\section{Conclusion}

Dans ce modeste ouvrage qu'implique le plus impressionnant et indomptable élément de la nature ; nous souhaitons avoir atteint l'objectif et réaliser notre dessein limité soit-il devant un phénomène quasi insaisissable de par son omniprésence et la complexité de sa constitution.

Avouons-le toutefois que le poète qui nous a confié ses méditations n'appartient guère à cette catégorie qui se livre facilement. Ce qui a rendu notre tâche ardue notamment lorsque nous nous retrouvons traqués par deux facteurs non négligeables : la briéveté d'un travail qui mériterait le titre d'une recherche universitaire et surtout le facteur chronologique.

Nous avons accepté le défi en comptant sur la mansuétude de nos lecteurs qui y retrouveront, nous le souhaitons ardemment, une idée sommaire mais largement persuasive de ce que représente la lumière chez un poète hermetique en dépit de sa simplicité patente. 
Nous n'avons rien laissé au hasard à commencer par la nature de cette lumière où se conjuguent l'abstrait et le concret dans une symbiose qui en dit long sur la fabuleuse et merveilleuse alchimie de son fonctionnement de par son omniprésence dans toute vie terrestre, planétaire et météorologique.

Comment élucider un imaginaire qui nous confie la présence de deux éléments inconciliables et paradoxaux dans un seul phénomène : l'élément aquatique et celui igné.

Le lecteur y retrouvera son compte sans prétendre avoir tout cerné dans notre trébuchant essai. Bien malin qui oserait, sans risque, aborder un thème si inextricable où une simple onde élecromagnétique, celle de la lumière se convertir en parole.

Cela fait partie de notre analyse à la recherche d'une solution pour ce phénomène qui nous fascine, nous intrigue avec toutes les embuches présumées de notre recherche.

Nous n'avons rien laissé au hasard et l'importance incontournable de cette lumière et son dynamisme qui nous inculque la vitalité, l'énergie et cette microbolante faculté ; la mobilité qui nous permet de franchir les barrières qui separent un être vivant d'une masse inerte et immibile.

Il sied de rendre un grand hommage, dans cette conclusion, à un poète qui nous a permis de palper 
l'insaisissable à la rencontre des mystères qui régissent la vie qui nous anime ; à partir de l'inflorescence des bourgeons d'une fleur jusqu'à l'apparition et la présence des phénomènes météorologiques. 
Marges

( ${ }^{1}$ - Jaccottet (Philippe) : La Semaison. Paris Gallimard. 1967.p.115

2- Jaccottet (Philippe) : La Semaison. Paris Gallimard. 1967.p.117

3-Ibid:p. 22

${ }^{4}$-Idem.

5- Idem.

${ }^{6}$ - Jaccottet (Philippe) : La Semaison. Paris Gallimard. 1967,p.22

${ }^{7}$ Ibid: p. 15

${ }^{8}$ - Jaccottet (Philippe) : La Semaison. Paris Gallimard. 1967.p15

${ }^{9}$ - Jaccottet (Philippe) : La Semaison. Paris Gallimard. 1967.p15

${ }^{10}$-Ibid: $\mathbf{p . 7 8}$

11 -Idem.

12 -Ibid: .p79

${ }^{13}$ - Jaccottet (Philippe) : La Semaison. Paris Gallimard. 1967.p.79

${ }^{14-}$ Ibid: p. 12

15 - Jaccottet (Philippe) : La Semaison. Paris Gallimard. 1967.p116 
${ }^{16}$-Ibid: P.34

17 - Jaccottet (Philippe) : La Semaison. Paris Gallimard. 1967.p95

${ }^{18}$-Ibid: p.70

19 - Ibid : .p38

20 - Jaccottet (Philippe) : La Semaison. Paris Gallimard. 1967.

P.47

${ }^{21}$-Idem.

${ }^{22}$-Ibid: p. 51

${ }^{23}$ - Idem.

${ }^{24}$ _ Jaccottet (Philippe) : La Semaison. Paris Gallimard. 1967.p.17

${ }^{25}$-Ibid: $\mathbf{p} .21$

${ }^{26}$ - Jaccottet (Philippe) : La Semaison. Paris Gallimard. 1967.p63

27_ Ibid: P.62

${ }^{28}$ - Ibid: .p127

${ }^{29}$ - Jaccottet (Philippe) : La Semaison. Paris Gallimard.

1967.p.127

${ }^{30}$ Idem

${ }^{31}$ Ibid: p.197

${ }^{32}$ Ibid:p.128 
33 -- Jaccottet (Philippe) : La Semaison. Paris Gallimard.

1967.p37

34 Idem.

35 - Jaccottet (Philippe) : La Semaison. Paris Gallimard. 1967:

p. 48

${ }^{36}$-Ibid: p.107

${ }^{37}$-Ibid: P. 60

${ }^{38}$--Ibid: $\mathbf{p} .80$

${ }^{39}$ - Jaccottet (Philippe) : La Semaison. Paris Gallimard. 1967.p.80

${ }^{40}$-Ibid: p.12

${ }^{41}$-Idem.

42 - Ibid: p.76

43 - Jaccottet (Philippe) : La Semaison. Paris Gallimard. 1967.

P.108

44 -Ibid: $\mathbf{p} .17$

${ }^{45}$ - Ibid: .p. 30

${ }^{46}$ - Jaccottet (Philippe) : La Semaison. Paris Gallimard. 1967

.p.33

${ }^{47}$-Ibid: P. 28

48 -- Jaccottet (Philippe) : La Semaison. Paris Gallimard. 1967.p55 
${ }^{49}$-Idem.

${ }^{50}$-Ibid: p. 96

${ }^{51}$-Ibid .p. 6

${ }^{52}$ - Jaccottet (Philippe) : La Semaison. Paris Gallimard. 1967p.66

${ }^{53}$-Idem.

54 -Idem.

55 - Jaccottet (Philippe) : La Semaison. Paris Gallimard. 1967.p.67

${ }^{56}$-Ibid: p. 68

57 -Idem.

${ }^{58}$-Ibid: p.31

59 - Jaccottet (Philippe) : La Semaison. Paris Gallimard.

1967.p.31

${ }^{60}$-Idem.

${ }^{61}$ - Jaccottet (Philippe) : La Semaison. Paris Gallimard. 1967

p.16

${ }^{62}$-Idem.

${ }^{63}$-Idem.

${ }^{64}$-- Jaccottet (Philippe) : La Semaison. Paris Gallimard. 1967.p.38

65 -Idem. 
${ }^{66}$-Ibid: P.22

${ }^{67}$ - Idem.

${ }^{68}$ - Jaccottet (Philippe) : La Semaison. Paris Gallimard. 1967.p.30

${ }^{69}$-Ibid: P.38

70 - Jaccottet (Philippe) : La Semaison. Paris Gallimard. 1967.p.33

${ }^{71}$-Idem.

72 -Ibid: P. 22

${ }^{73}$ Idem.

${ }^{74}$ - Jaccottet (Philippe) : La Semaison. Paris Gallimard. 1967.p.30

${ }^{75}$-Ibid: P.38

${ }^{76}$ - Ibid: p.33

77 - Jaccottet (Philippe) : La Semaison. Paris Gallimard. 1967.p.33

${ }^{78}$-Ibid: p.39

79 -Ibid: p.17

80 - Jaccottet (Philippe) : La Semaison. Paris Gallimard. 1967.p.18

81 -Ibid: p.19

${ }^{82}$ - Ibid; .p.30 
${ }^{83}$ - Jaccottet (Philippe) : La Semaison. Paris Gallimard. 1967.

p.31

${ }^{84}$ - Ibid: p.32

${ }^{85}$ - Idem.

${ }^{86}$ - Jaccottet (Philippe) : La Semaison. Paris Gallimard. 1967.p.59

${ }^{87}$-Ibid: p.34

${ }^{88}$ - Jaccottet (Philippe) : La Semaison. Paris Gallimard. 1967.p.34

${ }^{89}$ - Ibid: p.58

${ }^{90}$-Idem.

${ }^{91}$ - Jaccottet (Philippe) : La Semaison. Paris Gallimard. 1967.p.60

${ }^{92}$-Ibid: p. 55

93 - Jaccottet (Philippe) : La Semaison. Paris Gallimard. 1967.p.27

${ }^{94}$ - Ibid: p.29

95 -Ibid: p. 69

96 - Jaccottet (Philippe) : La Semaison. Paris Gallimard. 1967.p.39

${ }^{97}$-Ibid: p. 25 
98 -Idem.

${ }^{99}$-Idem.

100 - Jaccottet (Philippe) : La Semaison. Paris Gallimard.

1967.p.26

101 -Idem.

102 -Ibid: p. 27

103 - Jaccottet (Philippe) : La Semaison. Paris Gallimard. 1967.p.14

104 -Ibid: p.74

105 - Jaccottet (Philippe) : La Semaison. Paris Gallimard.

1967.p.74

106 - Idem.

107_ Jaccottet (Philippe) : La Semaison. Paris Gallimard.

1967.p.69

108 - Idem.

109 -Ibid: .p.63

110 - Jaccottet (Philippe) : La Semaison. Paris Gallimard. 1967.

pp.69,70

111 - Ibid: p.70

112 - Jaccottet (Philippe) : La Semaison. Paris Gallimard.

1967.p.12 
${ }^{113}$-Ibid: p.75

114 - Jaccottet (Philippe) : La Semaison. Paris Gallimard.

1967.p.75

115 -Ibid: .p.34

116 -Idem.

117 - Jaccottet (Philippe) : La Semaison. Paris Gallimard. 1967.p.35

118 -Idem.

119 -Idem.

120 -Idem.

121_ Jaccottet (Philippe) : La Semaison. Paris Gallimard.

1967.p.36

122 -Idem.

123 -Idem.

124 -Idem.

125 - Jaccottet (Philippe) : La Semaison. Paris Gallimard. 1967.p.1

${ }^{126}$-Ibid: $\mathbf{p} .38$

127 - Jaccottet (Philippe) : La Semaison. Paris Gallimard. 1967.

p.55

${ }^{128}$ - Ibid: p.72

${ }^{129}$ - Ibid: p. 61 
130 - Jaccottet (Philippe) : La Semaison. Paris Gallimard.

1967.p.61

131 -Ibid: p. 14

132 -Idem.

133 -Idem.

134 - Jaccottet (Philippe) : La Semaison. Paris Gallimard. 1967

p.15

${ }^{135}$ - Idem. 


\section{Bibliographie}

I- Corpus :

- Jaccottet (Philippe) : La Semaison. Paris Gallimard. 1967.

\section{II- Ouvrages sur Philippe Jaccottet :}

- Borel (Jacques) : Poésie et Nostalgie. Paris. Levrault. 1979.

- Clerval (Alain) : Philippe Jaccottet. Paris. Seghers. 1976.

- Hammer (Anne-Marie) : Philippe Jaccottet ou l'approche de l'insaisissable. Genève. 1982.

- Onimus (Jean) : Philippe Jaccottet et l'insaisissable évidence. Strasbourg. 1979.

- Richard (Jean Pierre) : Onze études sur la poésie moderne. Paris. Seuil. 1964.

- Seylaz Jean Luc) : Philippe Jaccottet, une poésie et ses enjeux. Lausanne. 1982.

Sitographie :

$\underline{\text {-Philippe Jaccottet - Poèmes de Philippe Jaccottet }}$ 
WWW.poemes.co ' philippe-jaccottet

-Cours sur Philippe Jaccottet - Jean-Michel Maulpoix

WWW.maulpoix.net > Jaccottetcours

-Cours sur la poétique de Philippe Jaccottet

WWW.Maulpoix.net > Jaccottetpoetique

$\underline{\text {-Philippe Jaccottet - lePetitLitteraire.fr }}$

www.lepetitlitteraire.fr > auteurs > philippe-jaccottet 